\title{
VPS35 Gene
}

National Cancer Institute

\section{Source}

National Cancer Institute. VPS35 Gene. NCI Thesaurus. Code C99645.

This gene is involved in protein transport 